\title{
INTELLIGENT STYLE TRANSFER OF FILM IMAGE BASED ON CYCLEGAN
}

\author{
Da Liu ${ }^{1,2}$, Huixin Wang ${ }^{1}$ and Bin $\mathrm{Wu}^{1}$ \\ ${ }^{I}$ College of Intelligence and Computing, Tianjin University, Tianjin 300350, PR China \\ ${ }^{2}$ China Research Institute of Film Science \& Technology, Beijing 100086, PR China
}

\begin{abstract}
With the development and application of deep learning, image style transfer has achieved an important breakthrough. It has been able to intelligently generate stylized natural, realistic and high-quality images, which can be applied to film screens to reduce the cost of artificial effects. This paper proposes an intelligent style transfer method of film image style based on CycleGAN. In view of the high spatial resolution, large size and rich details of high-tech format film images, we have modified the input layer of the network so that the network can better process film images, and replace the original GAN loss with WGAN loss to achieve a more stable training effect. As a result, style features can be better transformed between images, and at the same time, SSIM loss is added to the cycle consistency loss to enhance the recovery of images similarity to the original image and improve the quality of the generated image. Experiments show that this method is effective in processing the style transfer of film images, and can intelligently generate high-quality and natural realistic style transfer images.
\end{abstract}

\section{KEYWORDS}

High-tech Format Film, Image Style Transfer, Cycle-Consistent Generative Adversarial Networks (CycleGAN), Wasserstein GAN (WGAN), SSIM

\section{INTRODUCTION}

Film is the product of scientific and technological progress and an organic fusion of technology and art. High-tech is an important booster for the upgrading and high-quality development of the film industry. In recent years, artificial intelligence (AI), machine learning, deep learning, cloud computing, high performance computing (HPC), computer vision, computer graphics (CG/CGI), and other intelligence science $\&$ technology and the new generation of information communication technology (ICT) are more and more widely used in the cinema industry. The continuous deepening not only innovates the film production model, enriches the film production methods, significantly improves the level, ability and efficiency of film production, but also actively promotes the information construction and intelligent upgrade of the cinema industry to promote the transformation of the cinema industry into a high-tech industry upgrade. The cinema technology chain in all-digital era is shown in Figure 1.

Adapt to the developing needs of people in modern society for high-quality film content, based on $4 \mathrm{~K}$ (4096×2160), $8 \mathrm{~K}(8192 \times 4320)$, giant screen, high frame rate (HFR), high dynamic range (HDR), wide color gamut (WCG), visual special effects (VFX), immersive audio and other emerging audio-visual technologies, high-tech format film has become the development focus and application hotspot of the modern film industry. It has played a vital role in improving the audio-visual quality of film, movie watching experience and industrial efficiency. Therefore, vigorously developing high-tech format film, and promoting the intelligent application of high-tech format film production throughout the industry chain has become an important measure to promote fine film production and the construction of a strong film country. 


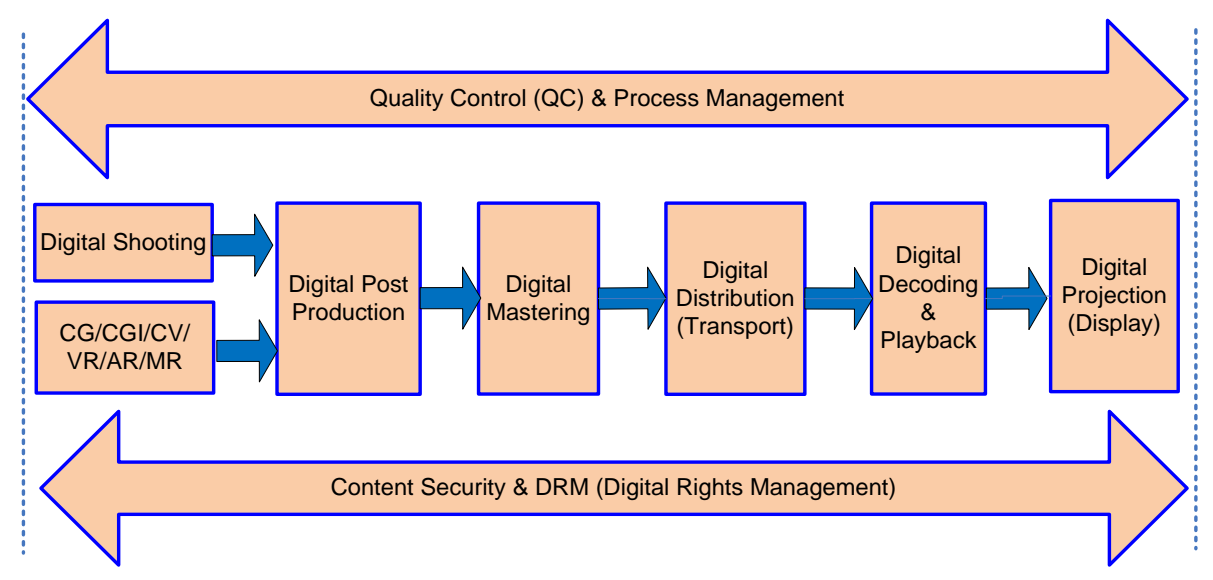

Figure 1. The cinema technology chain in all-digital era

Intelligentization is the basic trend and inevitable requirement for the development of modern film production. The intelligent transfer of styles for high-tech format film images is an effective means of modern film production, and has important application value for improving the level and efficiency of film production. Image style transfer refers to transferring the style of a reference image to another image while preserving the same structure and semantic information of the original image, which belongs to the research category of image translation, that is to realize cross-domain transformation of image. For example, a photo of a natural landscape is transform to the scene drawn by Van Gogh, as shown in Figure 2. Style transfer has a wide range of applications, and can be applied to film post production. Through image style transfer, the pictures taken by the camera can be transformed to other styles. The resulting picture will be more realistic and flexible.
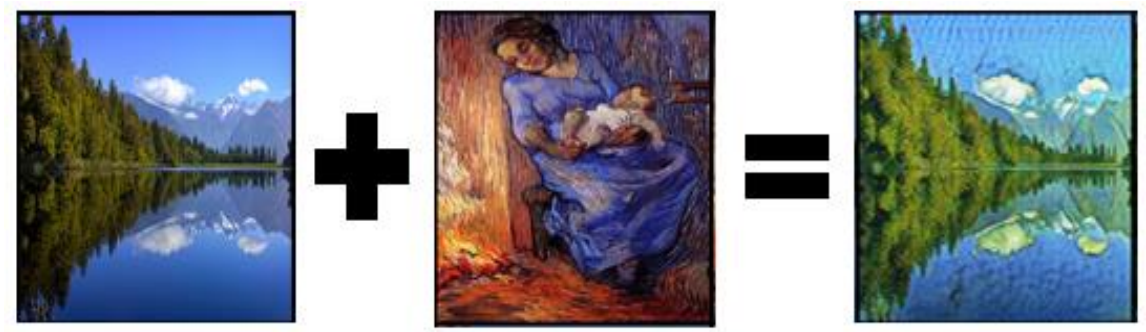

Figure 2. The natural scene transformed to Van Gogh's scene

So far, most existing style transfer methods are based on deep learning methods, which can achieve transfer between different scenes and different styles, and can obtain realistic and natural style transfer images. Convolution Neural Networks $(\mathrm{CNN})$, whose style transfer includes three main methods: single-model single-style, single-model multi-style, and single-model arbitrary style, but these methods will be limited and affected by the type and number of styles in the datasets. Generative Adversarial Networks (GAN), as a network trained by unsupervised learning small datasets, has great potential in the field of image style transfer, but training with only one GAN network may cause instability and result in poor final results. Cycle-Consistent Generative Adversarial Networks (CycleGAN) is composed of two symmetric GAN networks, especially for the field of style transfer to achieve better results. However, there is a clear difference between film images and general images. Most of the film images are high-resolution and large image sizes taken by the high-resolution professional film camera. The converted images need to take into account the artistry and true nature. The previous methods do not aim at this feature of film images, which will result in low quality of the generated results. The resulting image size is reduced, the resolution is also reduced, and it will be limited by storage and processing time, and the instability of network training will also be as a result, features cannot be transformed between images well.

In this paper, we propose a style transfer method of film images based on CycleGAN. According to the characteristics of film images, especially high-tech format film images, which based on emerging audio-visual technology, such as 4K, 8K, HFR, HDR, WCG, VFX, it can accurately transfer the style of reference images while saving the image content and details. Our method is based on CycleGAN's work on 
image style transfer. However, the image style transfer is directly performed by the CycleGAN method, for some parts of the source image, the original style cannot be transformed according to the reference image, and the resulting image quality is not high. Compared with general images, high-tech format film images has many technical performance advantages in the key technical indicators, such as spatial resolution, frame rate, quantization depth, dynamic range, color gamut. At the same time, the network of CycleGAN has some instability, which leads to bad final results. Our method has made some modifications to CycleGAN to make the model more suitable for film image style transfer. For the high-resolution, large-size and rich-detailed characteristics of film images, we have modified the input layer of the network on this basis so that the network can better process film images, while replacing the original GAN loss with WGAN loss to maintain the stability of training and adding structural similarity (SSIM) loss to the cycle consistency loss to improve the quality of the final generated image.

This paper proposes a method for film image style transfer based on the CycleGAN. On the one hand, this paper can realize the mapping of complex features to complex features, and change the style features of the source image according to the reference image. Meanwhile, the loss function used in this paper can ensure more stable results and high image quality results.

The main contributions of the paper are as follows:

(1) Propose a new high-tech format film image style transfer framework based on CycleGAN. For an input high-resolution, large-size and rich-detailed film image, the style can be transformed according to the reference image. The framework is end-to-end and requires no user interaction. We modify the input layer of the network so that the network can better process film images;

(2) Replace GAN loss with WGAN loss to ensure the stability of the model, so that the style features can be better transformed between the two images;

(3) The SSIM loss is added to the cycle consistency loss, and the quality of the generated image can be improved by adding the SSIM loss.

\section{RELATED WORK}

The problem of image style transfer is originally from the rendering of non-real images, which is closely related to the two fields of image texture modeling and image reconstruction. Texture is the key element of image style, texture characteristic is the characteristic that the image presents irregularity in the local area and regularity in the whole, and it mainly including roughness, directionality and contrast. The image texture transfer can be achieved by modeling the texture during image reconstruction. Texture modeling solves the problem of style features, mainly including parametric texture modeling methods based on statistical distribution and non-parametric texture modeling methods based on Markov random field (MRF); image reconstruction is to restore images based on features, mainly including online images optimized slow image reconstruction method and fast image reconstruction method based on offline model optimization. Gatys et al. (2016) proposed the first use of Convolution Neural Networks (CNN) for image style transfer. It is a pioneering work of image style transfer. The Gram matrix is used to model and extract the style of the image, and then use a slow image reconstruction algorithm. The Gram matrix is made close to the Gram matrix of the style graph, the high-level features of the VGG network are close to the features of the content graph, and the final reconstructed image contains both image content and image style. However, because the feature extraction is done in the high-level CNN feature space, some low-level information will be lost and some distortions will appear in the result. Li et al. (2017) proposed a MRF loss to replace the Gram loss, and split the style image and the reconstructed image into patches. For the patch of the reconstructed image to find the closest style patch, it can also work well when the style image is an image close to the content image retain structural information. The above method is based on the slow image reconstruction method for image style transfer, which is slow and consumes resources. There are still certain limitations in practical applications. The fast image style transfer algorithm uses the fast image reconstruction method optimized by the offline model to reconstruct the stylized results, and the forward network is pre-trained to solve the problem of large amount of calculation and slow speed. Li and Wand (2016) and Johnson et al. (2016) use the forward network to learn a style, and use the gram matrix for style modeling, but this single-model single-style algorithm requires training a model for each style, which is not particularly conducive to use. The work (Li \& Wand, 2016, October) on the basis of the trained style network, an affine transformation is performed 
on the Instance Normalization layer, namely Conditional Instance Normalization (CIN), which corresponds the parameters of the CIN layer to the style. For a new style, only the parameters need to be retrained to achieve a single multi-style effects of the model. In the CNN feature space, Zhang and Dana (2017) find the style patch that matches the content patch to exchange, and then use the fast image reconstruction algorithm to quickly rebuild according to the feature map after the exchange, but the time overhead is large. Inspired by the CIN layer, Ulyanov et al. (2017) proposed the Adaptive Instance Normalization (AdaIN). The input of AdaIN is the image style and content features extracted through VGG, which is trained on large-scale style images and content images, so that AdaIN can directly transfer the content image into different styles. The shortcomings of this method are also obvious. This data-driven approach leads to stylization effects that are very related to the type and number of styles in the training data set. Dosovitskiy and Brox (2016) have generated impressive results based on generative adversarial networks (GAN), but the training process is not very stable. However there have been some GAN-based works (Li et al., 2018; Verma \& Smith, 2017) in the field of image style transfer that have achieved good results.

Completing image style transfer based on GAN is a promising direction. GAN and its derivative model have important application value for the intelligent post-production of movies, and have great significance for the intelligent upgrading of cinema industry. Generative Adversarial Networks (GAN), proposed by Goodfellow et al. (2014), is a novel unsupervised learning method, which based on Zero-Sum Game and Nash Equilibrium theories. The core parts of GAN are generator and discriminator, also known as generative model and discriminative model. GAN can solve the training problem of small data sets, and does not need to calibrate the data. It can be learned directly on the network. Radford et al. (2015) introduced a convolutional neural network, called deep convolution generative adversarial network (DCGAN). It changed the network architecture of GAN to make the network training more stable. Although the network has a good architecture, it does not really solve the GAN training stability problem. Wasserstein GAN (WGAN) proposed by Arjovsky et al. (2017) has made progress in GAN stability training. The generative model of the traditional GAN is to convert random noise into a picture, but in the image style transfer, we need to convert the image into a image, so this time we must use the image as the input of generative model, and generative model is to learn a mapping. However, training using only one GAN is unstable, which may cause all photos to be mapped to mode collapse of the same picture. At this time, CycleGAN (Zhu et al., 2017) was proposed to solve this problem. CycleGAN is a combination of two GANs, which can realize the conversion between unpaired images, especially suitable for image style transfer.

\section{METHOD}

The input of our network is mainly two images: one input image is a large-scale high-resolution film image, and the other input is a reference image with special style. Through the adversarial networks, the style of the input image is converted into the style of the reference image, while maintaining the realism and naturalness and high quality of the resulting style transfer image.

The network framework of this paper is shown in Figure 3. Our network is based on the CycleGAN network, which is composed of two unidirectional symmetric GAN models. These two networks form a ring network. It can be seen from the figure that the network is composed of two sets of generators and two sets of discriminators, and information is shared between them. Input a pair of source image $\mathrm{X}$ and reference image $\mathrm{Y}$, the input image $\mathrm{X}$ is passed to the first generator $\mathrm{G}$, and then the newly generated image fake_Y is passed to another generator F, the fake_Y is restored to get rec_X. Similarly, the training process of the source image $\mathrm{Y}$ can be obtained. The input image $\mathrm{Y}$ is passed to the generator $\mathrm{F}$, and then the newly generated image fake_X is passed to another generator $\mathrm{G}$ to restore fake_X. The discriminator $\mathrm{D}$ is used to discriminate whether the image is the original image or the generated image, DX judges the authenticity of the source image $\mathrm{X}$ and fake_X, and DY judges the authenticity of the source image $\mathrm{Y}$ and fake_Y.

\subsection{Network Architectures}

Generator Network. For the generator network, the encoder mainly includes 3 convolution neural networks $(\mathrm{CNN})$, the convolution kernel is $3 \times 3$, and the step size is 2 ; the converter includes 6 residual networks, which are composed of 2 convolution layers, and can guarantee that the previously entered information can 
be input into the following network. It can reduce the difference between the previous input information and the subsequent input information, to ensure that the details of the output image of the network remain intact; the decoder is mainly composed of 2 deconvolution layers and 1 convolution layer Composition, the convolution kernel of the deconvolution layer is $3 \times 3$, the step size is 2 , and the convolution kernel of the convolution layer is $7 \times 7$. When inputting a $1024 \times 1024 \times 3$ image, after convolving the image through 2 convolution layers, 1024 output features with a size of $256 \times 256$ can be obtained. After the residual network processing, the $256 \times 256$ output features of the previous step can be passed to the converter to obtain feature vectors of the same size.

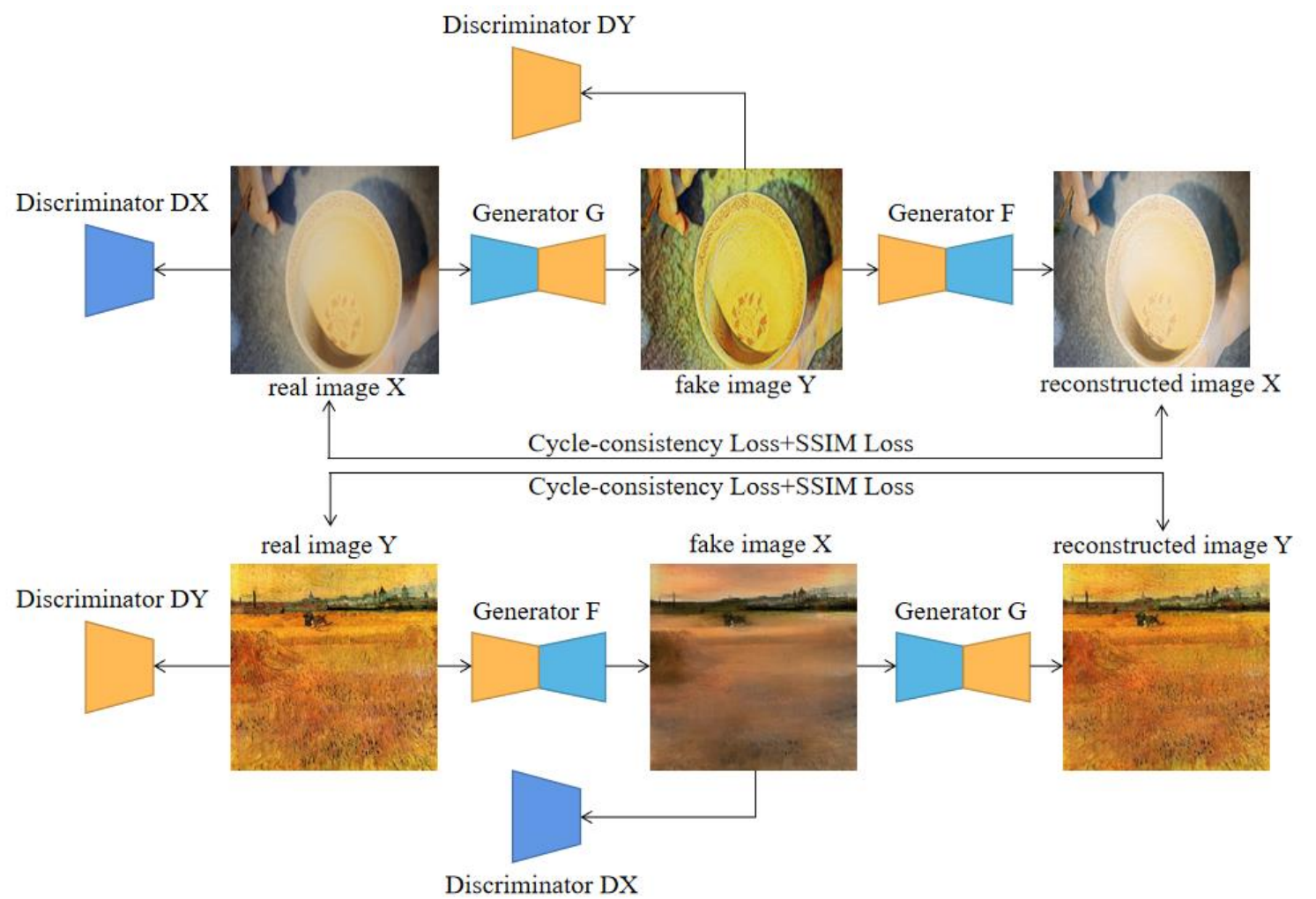

Figure 3. Our network framework

Discriminator Network. The discriminator network needs to identify the real image or the generated image by extracting image features. Therefore, this paper uses a convolution neural network (CNN) as a discriminator. For the discriminator network, we mainly use 4 fully convolutional layers and a 1D output convolution layer to implement the discrimination function. Use $70 \times 70$ PatchGAN to identify whether $70 \times 70$ overlapping image blocks are real or fake. This block-level discriminator structure has fewer parameters than the full-image discriminator, and can be applied to images of any size in a fully convolutional manner.

\subsection{Loss}

Avoid color images as the proceedings will be printed in black and white. The goal of this paper is to learn the mapping function between two domains $X$ and $Y$ given training samples, where $x_{i} \in X$ and $y_{j} \in Y$. For simplicity, we omit the subscripts $\mathrm{i}$ and $\mathrm{j}$. DX and DY are two adversarial discriminators. DX is used to distinguish the image $\{\mathrm{x}\}$ from the transformed image $\{\mathrm{F}(\mathrm{y})\}$, and $\mathrm{DY}$ is to distinguish $\{\mathrm{y}\}$ from $\{\mathrm{G}(\mathrm{x})\}$. The objective function consists of three parts: the first is the WGAN loss, which is used to match the distribution of the generated image with the data distribution in the target domain to maintain the stability of training; the second is the loss of cycle consistency loss and structural similarity (SSIM) loss, cycle consistency loss can be used to prevent contradictions when learning $G$ and F mapping relationships, SSIM loss is used to strengthen the similarity between the restored image and the original image and maintain the quality of the 
generated image; the third is a loss of identity that can maintain the color composition between input and output.

WGAN Loss. For the traditional GAN network, there are problems such as training difficulties, the loss of the generator and discriminator cannot indicate the training process, and the lack of diversity in the generated samples. Wasserstein GAN (WGAN) (Gulrajani et al., 2017) solves the problem of GAN training instability, no longer need to carefully balance the training level of the generator and discriminator, and basically solve the problem of mode collapse, ensuring the diversity of generated samples. From our test results, we can see that in some test results, the use of GAN loss will make some styles of the reference image unable to be transferred to the source image. This is because the standard discriminator loss uses cross-entropy loss, which causes the gradient to disappear. In order to solve this problem, we have improved according to WGAN: the loss of the generator and discriminator no longer takes the log function, the output of the discriminator is no longer a probability, so the last layer of the discriminator is removed sigmoid layer; after the discriminator is updated every time, the absolute value of the parameters is truncated to no more than a fixed constant c; use Root Mean Square Prop (RMSProp) or Stochastic Gradient Descent (SGD) instead of using momentum-based optimization algorithms (including momentum and Adam).

Cycle Consistency Loss and SSIM Loss. When lossy compression is performed on an image, or an image has noise, distortion, etc., Wang et al. (2004) proposed a metric to measure the similarity between the reconstructed image and the original image: Structural Similarity (SSIM) index. SSIM loss is a loss function driven by perception. The goal is to allow the network to learn to generate visually pleasing images, and SSIM loss has been shown to improve the quality of generated images. In order to improve the quality of the final generated image on the basis of SSIM loss, we chose MS-SSIM loss (Wang et al., 2003). MS-SSIM loss considers SSIM loss on the M scale as follows:

$$
M S-\operatorname{SSIM}(x, y)=\left[l_{M}(x, y)^{\alpha_{M}} \prod_{j=1}^{M}\left[c_{j}(x, y)\right]^{\beta_{j}}\left[s_{j}(x, y)\right]^{\gamma_{j}}\right.
$$

Where the luminance is $1(x, y)$, the contrast is $\mathrm{c}(\mathrm{x}, \mathrm{y})$, and the structure is $\mathrm{s}(\mathrm{x}, \mathrm{y})$,

$$
\begin{aligned}
& l(x, y)=\frac{2 \mu_{x} \mu_{y}+c_{1}}{\mu_{x}^{2}+\mu_{y}^{2}+c_{1}} \\
& \mathrm{c}(x, y)=\frac{2 \sigma_{x} \sigma_{y}+c_{1}}{\sigma_{x}^{2}+\sigma_{y}^{2}+c_{1}} \\
& \mathrm{~s}(x, y)=\frac{\sigma_{x y}+c_{3}}{\sigma_{x} \sigma_{y}+c_{3}}
\end{aligned}
$$

For convenience, we set $\alpha_{\mathrm{M}}$ and $\beta_{\mathrm{j}}$ to 1 , and the loss calculated at the center pixel can be approximated to estimate the loss of patch P:

$$
\mathrm{L}_{\text {MS-SSIM }}(P)=1-\operatorname{MS}-\operatorname{SSIM}(\tilde{p})
$$

We added SSIM loss to the cycle consistency loss to enhance the similarity between the reconstructed image and the original image.

Identity Loss. In the process of image generation, due to the influence of the generated image, the mapping of color features may be changed, and the color composition between the reconstruction image and the source image changes greatly, making people visually feel the difference between the source image and the reconstruction image larger. Therefore, the identity loss (Zhu et al., 2017) is added to make the color composition of the source image and the reconstruction image consistent. The loss of identity is as follows:

$$
\mathrm{L}_{\text {identity }}(G, F)=\mathrm{E}_{y \sim P_{\text {data }}(y)}\left[\|G(y)-y\|_{1}\right]+\mathrm{E}_{x \sim P_{\text {data }}(x)}\left[\|G(x)-x\|_{1}\right]
$$

We integrate different loss functions:

$$
L_{\text {loss }}=\lambda L_{W G A N}+\alpha L_{c y c+\mathrm{MS}-S S I M}+\beta L_{\text {identity }}
$$




\section{EXPERIMENTS}

Training. For all experiments, we set $\lambda=10, \alpha=1, \beta=0.1$ in formula (7). All networks are trained from scratch, with a learning rate of 0.0002 . One epoch means that all samples in the training set have been experienced once. We maintain the same learning rate in the first 100 epochs, and linearly decay the rate to zero in the next 100 epochs.

Implementation Details. The requirements of the experimental environment in this paper are as follows: The processor is Intel Xeon, the graphics card used is Quadro M4000, and it runs on Linux Ubuntu 16.4 operating system. This paper uses the PyTorch framework, programming in Python, the number of iterations is set to 100 .

Dataset. For reference images, a total of 2076 images in four styles including Vangogh, Monet, Cezanne, and Ukiyoe were collected (see Figure 4). Considering that the film images are mostly images in natural scenes, as shown in Figure 5, we collected a total number of 6015 images with an image size of $1024 \times 1024$ for experiments.
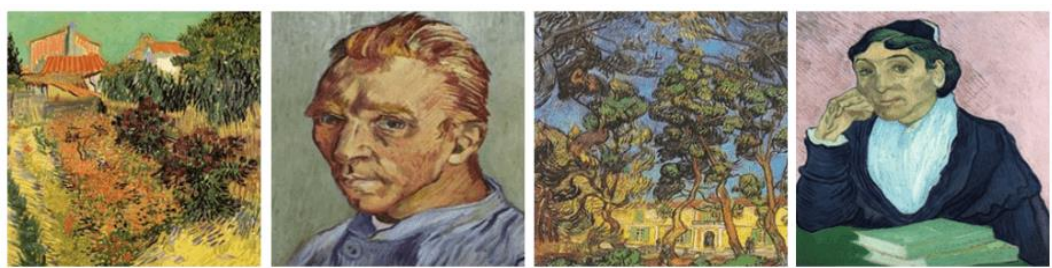

(a) Vangogh Style
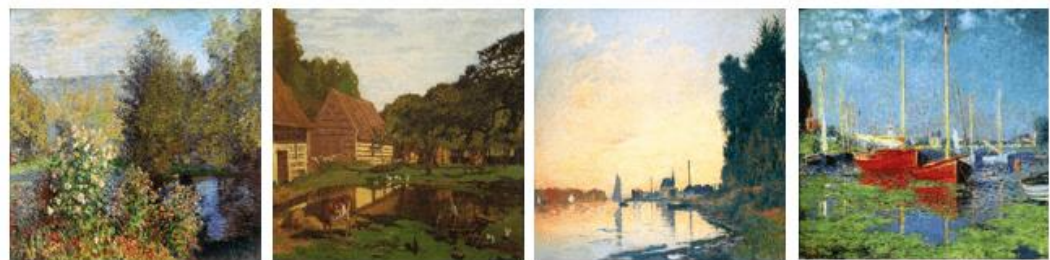

(b) Monet Style
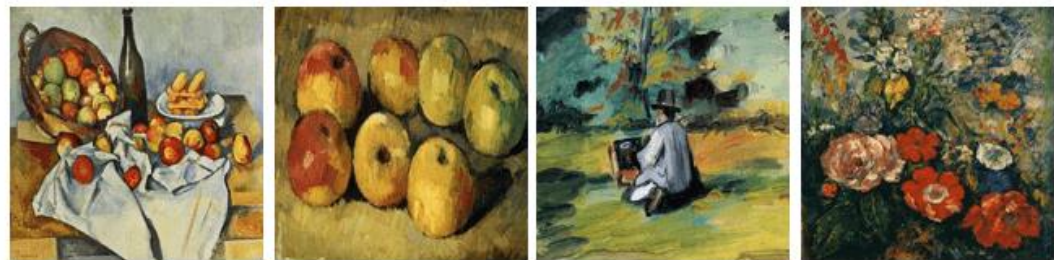

(c) Cezanne Style
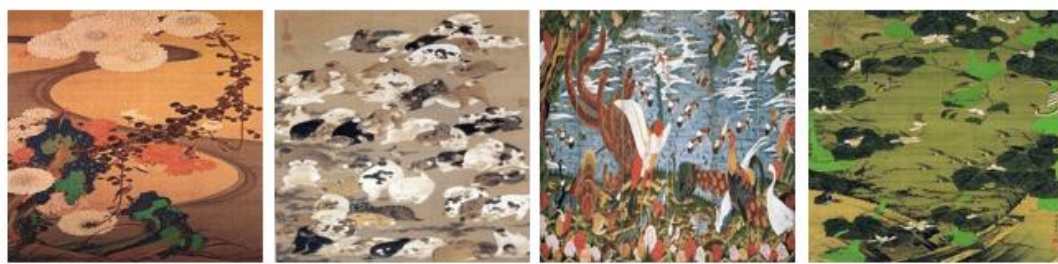

(d) Ukiyoe Style

Figure 4. Example of style images 

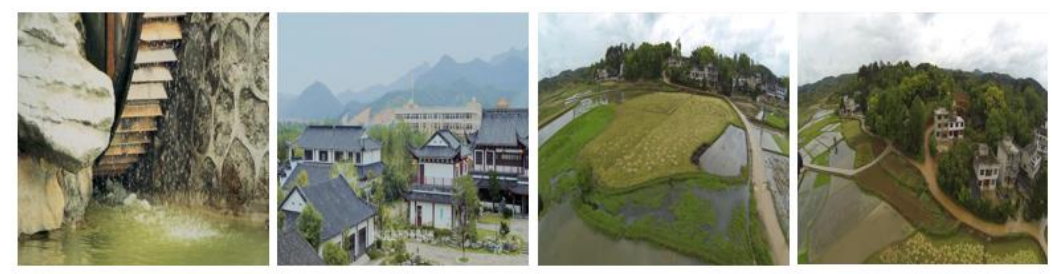

Figure 5. Example of film images

Quantitative Results. We trained our model on four datasets, and transferred the style the source image to generate a new image, so that the generated images have the style of Vangogh, Cezanne, Ukiyoe and Monet. We show the results in Figure 6. For example, the generated Vangogh style image seems to be Vangogh's drawing of this picture in reality. Compared with the source image, it keeps the content and details of the source image. Compared with the style image, it inherits the style information of the style image, including texture and color. The image obtained by our method keeps a good balance in style and content.

We also compare our method with the methods of Gatys et al. and Johnson et al. in Figure 7. The methods of Gatys et al. and Johnson et al. transfer the image style information such as color and texture of the style image to the source image, but at this time, the content and details of the source image are kept less. Although the images generated by these two methods can generate images with corresponding styles, they will make people feel unnatural and unreal, and it can be clearly seen that they are generated artificial false images. But the image generated by our method keeps more details and contents of the source image after the style is transferred, and the generated image is just like the real image generated in this style. The methods of Gatys et al. and Johnson et al. will produce uneven color distribution. For example, the second image in Figure 7, the method of Gatys et al. has abrupt green areas and the method of Johnson et al. has sudden red areas, but our method will not produce this kind of unnatural color transfer error. And the results by Gatys et al. and Johnson et al. will lose some details of the source image, for example, for the first image, the results obtained by Johnson et al. lose the sky information of the source image. For the fourth image, the results obtained by Gatys et al. lose the exposed land distribution information of the source image, but our method can keep more details of the source image.

Especially for the style transfer of film images, in practical application, more attention is paid to the natural authenticity of the image after style transfer. The image after style transfer needs to make the audience feel real, which is different from the general style transfer. Compared with Gatys et al. and Johnson et al., our method keeps the content and details of the source image as much as possible while performing style transfer, and generates a more natural and realistic style transfer image. Our method is more suitable for style migration of film images.

\section{CONCLUSION}

We proposed a method for intelligent style transfer of film images based on CycleGAN. In view of the high-resolution and large size of high-tech format film images, as well as the instability of CycleGAN training and the low quality of the generated images, we modified the input layer of the network to train the network, and replaced the original GAN loss with the WGAN loss which makes the training process more stable, and the style features can better migrate between images. The SSIM loss is added to the cycle consistency loss to improve the quality of the generated image. The method in this paper can be based on the characteristics of film images, according to the reference image to transform the style of the source image to generate high-quality, natural and realistic images. 


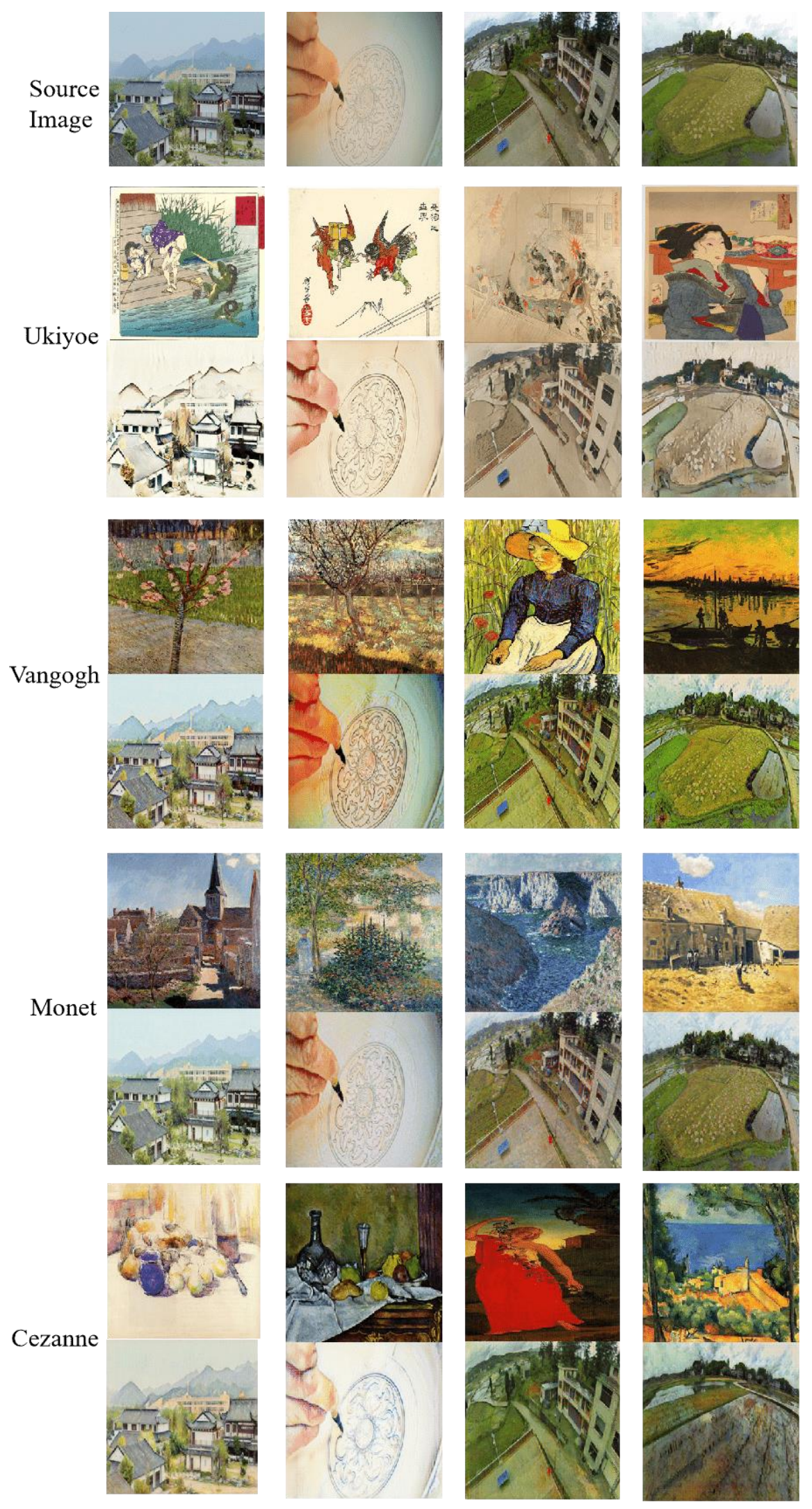

Figure 6. Qualitative results 

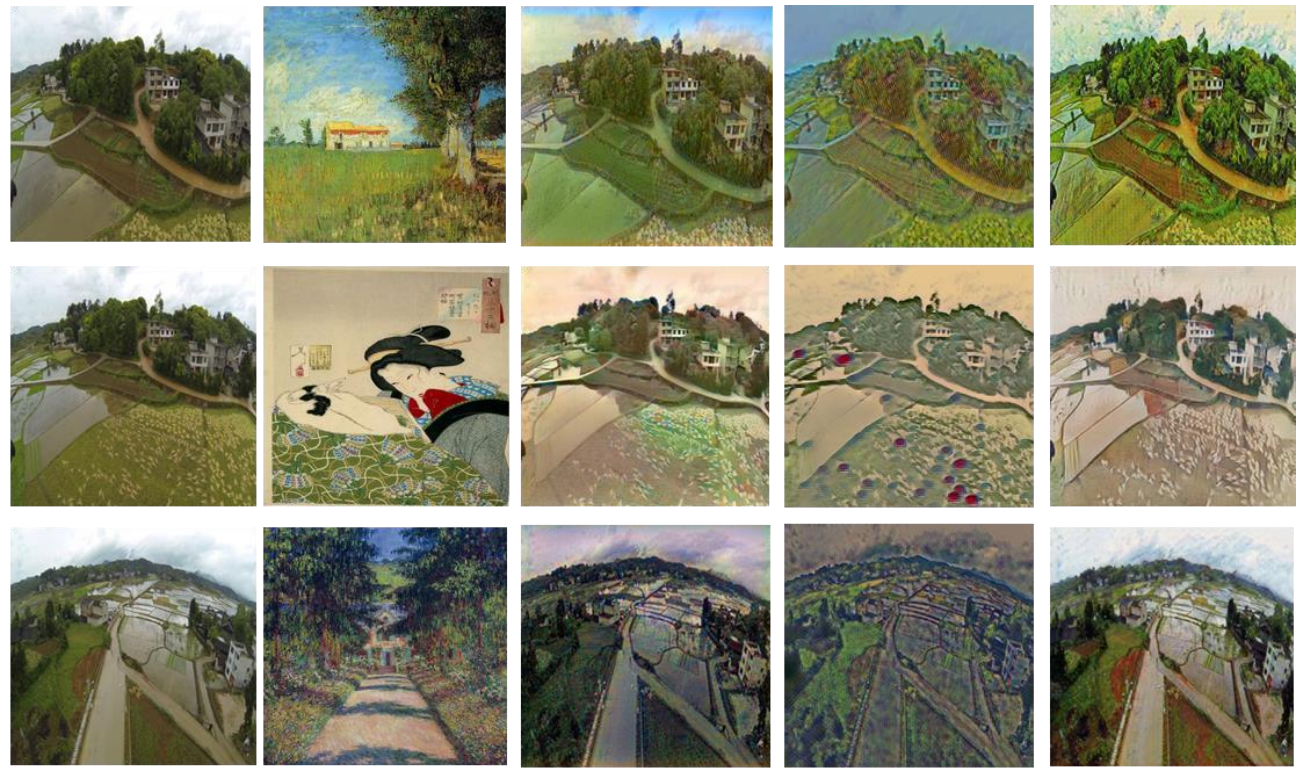

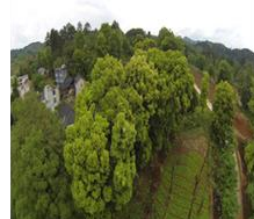

(a)Source image

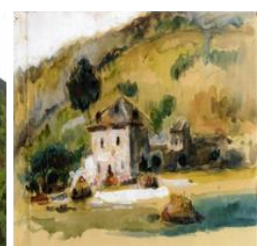

(b)Style image

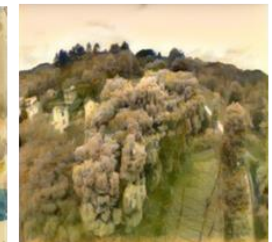

(c)Gatys et al.

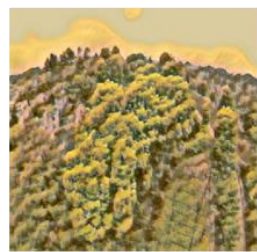

(d)Johnson et al.

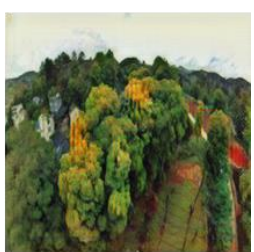

(e)our results

Figure 7. Example style transfer results. Comparison with Gatys et al. and Johnson et al.

\section{REFERENCES}

Arjovsky, M., Chintala, S., \& Bottou, L. (2017). Wasserstein gan. arXiv preprint arXiv:1701.07875.

Digital Cinema Initiatives (2018). Digital cimena system specification Version 1.3.

Digital Cinema Initiatives (2019). DCI statement on technology evolution in digital cinema.

Dosovitskiy, A., \& Brox, T. (2016). Generating images with perceptual similarity metrics based on deep networks. In Advances in neural information processing systems (pp. 658-666).

Gatys, L. A., Ecker, A. S., \& Bethge, M. (2016). Image style transfer using convolutional neural networks. In Proceedings of the IEEE conference on computer vision and pattern recognition (pp. 2414-2423).

Goodfellow, I., Pouget-Abadie, J., Mirza, M., Xu, B., Warde-Farley, D., Ozair, S., Courville, A., \& Bengio, Y. (2014). Generative adversarial nets. In Advances in neural information processing systems (pp. 2672-2680).

Gulrajani, I., Ahmed, F., Arjovsky, M., Dumoulin, V., \& Courville, A. C. (2017). Improved training of wasserstein gans. In Advances in neural information processing systems (pp. 5767-5777).

Johnson, J., Alahi, A., \& Fei-Fei, L. (2016, October). Perceptual losses for real-time style transfer and super-resolution. In European conference on computer vision (pp. 694-711). Springer, Cham.

Li, C., \& Wand, M. (2016). Combining markov random fields and convolutional neural networks for image synthesis. In Proceedings of the IEEE Conference on Computer Vision and Pattern Recognition (pp. 2479-2486).

Li, C., \& Wand, M. (2016, October). Precomputed real-time texture synthesis with markovian generative adversarial networks. In European conference on computer vision (pp. 702-716). Springer, Cham.

Li, S., Xu, X., Nie, L., \& Chua, T. S. (2017, October). Laplacian-steered neural style transfer. In Proceedings of the 25th ACM international conference on Multimedia (pp. 1716-1724).

Li, Y., Liu, M. Y., Li, X., Yang, M. H., \& Kautz, J. (2018). A closed-form solution to photorealistic image stylization. In Proceedings of the European Conference on Computer Vision (ECCV) (pp. 453-468). 
Radford, A., Metz, L., \& Chintala, S. (2015). Unsupervised representation learning with deep convolutional generative adversarial networks. arXiv preprint arXiv:1511.06434.

Ulyanov, D., Vedaldi, A., \& Lempitsky, V. (2017). Improved texture networks: Maximizing quality and diversity in feed-forward stylization and texture synthesis. In Proceedings of the IEEE Conference on Computer Vision and Pattern Recognition (pp. 6924-6932).

Verma, P., \& Smith, J. O. (2018). Neural style transfer for audio spectograms. arXiv preprint arXiv:1801.01589.

Wang, Z., Bovik, A. C., Sheikh, H. R., \& Simoncelli, E. P. (2004). Image quality assessment: from error visibility to structural similarity. IEEE transactions on image processing, 13(4), 600-612.

Wang, Z., Simoncelli, E. P., \& Bovik, A. C. (2003, November). Multiscale structural similarity for image quality assessment. In The Thrity-Seventh Asilomar Conference on Signals, Systems \& Computers, 2003 (Vol. 2, pp. 1398-1402). Ieee.

Zhang, H., \& Dana, K. (2017). Multi-style generative network for real-time transfer. arXiv preprint arXiv:1703.06953.

Zhu, J. Y., Park, T., Isola, P., \& Efros, A. A. (2017). Unpaired image-to-image translation using cycle-consistent adversarial networks. In Proceedings of the IEEE international conference on computer vision (pp. 2223-2232). 